\title{
Transvenous shock-only implantable cardioverter defibrillator for tricuspid valve atresia after an atrio-pulmonary Fontan surgery
}

\author{
Toyohara Keiko ${ }^{1}$, Daigo Yagishita ${ }^{1}$, Yoshimichi Kudo ${ }^{1}$, Tomomi Nishimura ${ }^{1}$, Daiji \\ Takeuchi $^{1}$, Yasuko Tomizawa ${ }^{1}$, and Morio Shoda ${ }^{1}$
}

${ }^{1}$ Tokyo Women's Medical University

October 5, 2020

\begin{abstract}
A 42-year-old woman with tricuspid atresia who underwent a Fontan surgery (atrio-pulmonary connection) was admitted to our hospital due to symptomatic ventricular tachycardia. A defibrillation lead was implanted in a distal site of a coronary vein since there was no usual entry to the ventricle. Ventricular pacing was impossible due to the high threshold, however, good sensing was obtained. Three years later, she felt palpitations and a subsequent shock therapy while climbing stairs. The cardioverter data showed that an appropriate cardioversion therapy successfully converted ventricular tachycardia to normal rhythm.
\end{abstract}

\section{Hosted file}

20201002 APC_CS_ICD_JCE.pdf available at https://authorea.com/users/364389/articles/484839transvenous-shock-only-implantable-cardioverter-defibrillator-for-tricuspid-valveatresia-after-an-atrio-pulmonary-fontan-surgery

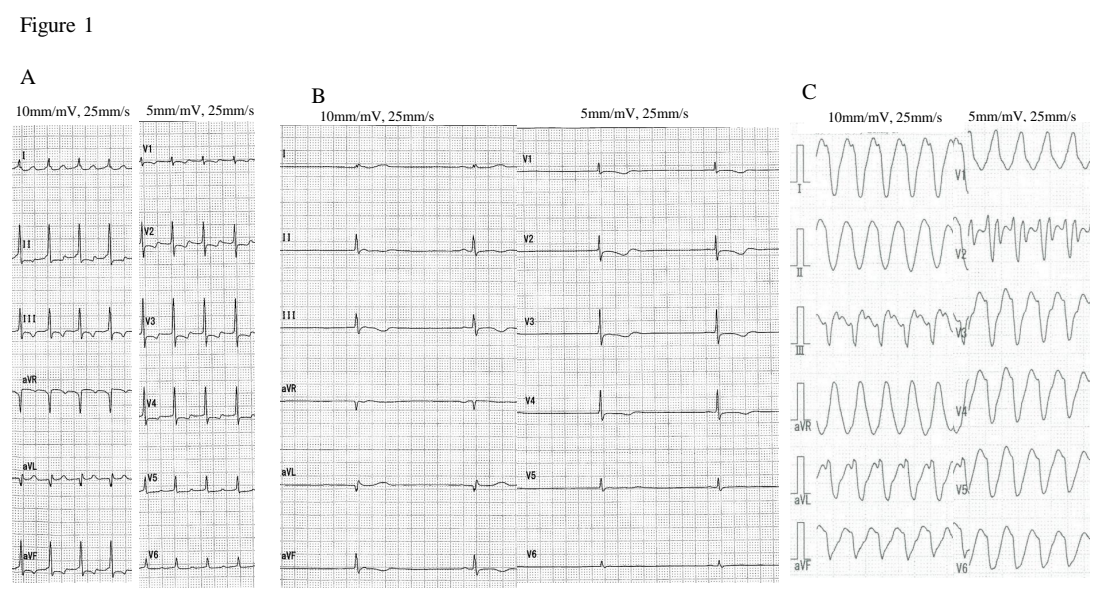

